\title{
A Novel Network Modeling and Evaluation Approach for Security Vulnerability Quantification in Substation Automation Systems
}

\author{
Jongbin $\mathrm{KO}^{\dagger}$, Seokjun $\mathrm{LEE}^{\dagger}$, Yong-hun LIM ${ }^{\dagger \dagger}$, Seong-ho JU ${ }^{\dagger \dagger}$, Nonmembers, and Taeshik SHON ${ }^{\dagger \mathrm{a})}$, Member
}

\begin{abstract}
SUMMARY With the proliferation of smart grids and the construction of various electric IT systems and networks, a next-generation substation automation system (SAS) based on IEC 61850 has been agreed upon as a core element of smart grids. However, research on security vulnerability analysis and quantification for automated substations is still in the preliminary phase. In particular, it is not suitable to apply existing security vulnerability quantification approaches to IEC 61850-based SAS because of its heterogeneous characteristics. In this paper, we propose an IEC 61850based SAS network modeling and evaluation approach for security vulnerability quantification. The proposed approach uses network-level and device groupings to categorize the characteristic of the SAS. In addition, novel attack scenarios are proposed through a zoning scheme to evaluate the network model. Finally, an MTTC (Mean Time-to-Compromise) scheme is used to verify the proposed network model using a sample attack scenario. key words: IEC 61850, substation automation system, security vulnerability quantification, smart grid, IEC 62351, CVSS, MTTC
\end{abstract}

\section{Introduction}

In smart grids, a substation system is upgraded to a substation automation system (SAS) by applying IEC 61850. IEC 61850-based SAS combines a legacy computer network protocol (Ethernet, TCP/IP, etc.) and a substation-exclusive protocol (GOOSE, MMS, SV, etc.). This has made the supervision and control of SASs externally through the main control center of smart grids more efficient and easy. However, this implies that the security advantage of isolation from external networks would be lost. Therefore, there is a need for a new security technology paradigm to address new threats and vulnerabilities to IEC 61850-based SASs.

The first consideration in this new security paradigm will be the accurate understanding of the security vulnerabilities in the new system. Security vulnerability quantification schemes estimate the vulnerability of a system by checking susceptibilities. Thus, this is a suitable area for primary research.

The goal of an IEC 61850-based SAS network is to standardize message exchange between different devices for substation control and automation; hence, its characteristics and structure differ from commercial computers. Moreover, intelligent electronic devices (IEDs), the principal devices connected by the IEC 61850 standard, have techni-

Manuscript received January 16, 2013.

Manuscript revised April 25, 2013.

$\dagger$ The authors are with the Division of Computer Engineering, Ajou University, Suwon, Korea.

${ }^{\dagger \dagger}$ The authors are with the Korea Electric Power Corporation (KEPCO) Research Institute, Daejeon, Korea.

a) E-mail: tsshon@ ajou.ac.kr

DOI: 10.1587/transinf.E96.D.2021 cal and functional differences from standard computers and it constructs large scale network using various protocols. Thus, existing security vulnerability quantification schemes for legacy computer networks are unsuitable for IEC 61850based SASs. Therefore, it is necessary to develop a novel network modeling approach for quantifying security vulnerability.

In this paper, we propose a network modeling approach with attack scenarios for security vulnerability quantification of IEC 61850-based SAS. We evaluate the proposed approach by applying a security vulnerability quantification scheme-MTTC.

In Sect. 2, we will present the existing vulnerability quantification schemes and discuss their unsuitability for an IEC 61850-based SAS. In Sect. 3, the SAS network architecture of IEC 61850 will be described. In Sect. 4, the proposed network modeling approach for quantifying security vulnerability will be presented. It includes network-level and device groupings for solving the complexity of the IEC 61850 network structure. We will also propose attack scenarios that use multiple attack routes based on a zoning scheme. In Sect. 5, we will show our evaluation results. Finally, we will conclude the paper and discuss future work in Sect. 6 .

\section{Existing Vulnerability Quantification Schemes for SAS}

\subsection{Common Vulnerability Scoring System}

The Common Vulnerability Scoring System (CVSS) [3] was developed by a group of corporations including CERT/CC, Cisco, DHS/MITRE, eBay, IBM, and Microsoft) to create a standardized, open vulnerability scoring framework. CVSS offers an objective and formal procedure to the vendors and users (e.g., security administrator) for evaluating the vulnerability of the target system.

CVSS has three metric groups-base, temporal, and environmental-that consist of several metrics such as access vector, access complexity, authentication, confidentiality impact, integrity impact, exploitability, and target distribution.

- Base: represents the intrinsic and fundamental characteristics of vulnerabilities that are constant over time and user environments.

- Temporal: represents the characteristics of vulnerabili- 


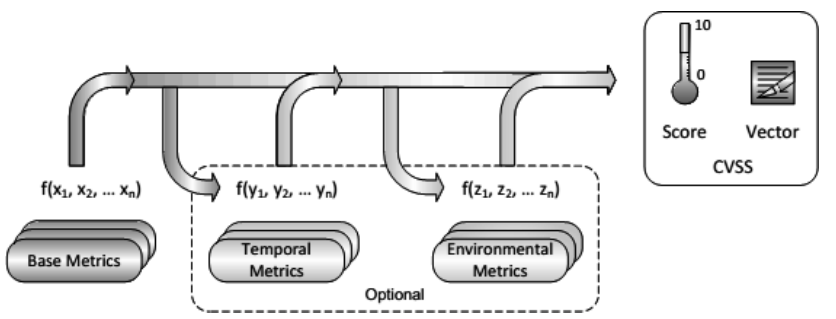

Fig. 1 CVSS metrics and equations [1].

ties that change over time but not among user environments.

- Environmental: represents the characteristics of vulnerabilities that are relevant and unique to a particular user environment.

CVSS uses the base group to define and communicate the fundamental characteristics of vulnerabilities. The temporal and environmental groups are selectively used to provide contextual information that reflect risks particular to their unique environment more accurately.

When the base metrics are assigned values, the base equation calculates a score ranging from 0 to 10 and creates a vector, as illustrated in Fig. 1. The vector facilitates the "open" nature of the framework. It is a text string that contains the values assigned to each metric, and it is used to communicate exactly the derivation of the score for each vulnerability. Therefore, the vector should always be displayed with the vulnerability score [1].

\subsection{Limitation of CVSS on IEC 61850-Based SAS}

(A) Dependence on the vulnerability database

CVSS can score the unveiled vulnerabilities of a system. CVSS uses a vulnerability database such as the Common Vulnerability Exposure (CVE) [2] or National Vulnerability Database (NVD) [3] for scoring the system vulnerabilities. However, security vulnerabilities have fluid characteristics that can be changed or recreated by developing technologies or changing environment. Therefore, because of its dependence on a database, CVSS has limitations in its ability to deal effectively with new vulnerabilities on SAS devices such as IEDs.

(B) Essential differences of the target

IEC 61850 [4] is the standard used to exchange messages between heterogeneous electric devices to control and automate substations. An IED is the principal device connected using the IEC 61850 standard. IEDs have significant functional and structural differences from general PCs; these differences make CVSS, which calculates the vulnerability of a standard computer system and network, unsuitable for IEC 61850-based SAS.

\subsection{Other Schemes for SAS and SCADA Control Systems}

A recent work specializing in IEC 61850-based SAS proposed a scheme based on a security metric for IEDs [5]. The metric assigns a score to each IED by identifying the IED's vulnerabilities and checking their countermeasures. Then, it collects the scores of all the IEDs and calculates the final vulnerability score.

Many studies have focused on the Supervisory Control and Data Acquisition (SCADA) system as the target of vulnerability quantification. Some papers try to apply CVSS or improved CVSS to SCADA [6], [7]. These ideas have the same unsolved problems mentioned above. Other papers have proposed new vulnerability quantification ideas for SCADA systems using an attack tree or attack graph [8], [9]. Generally, the SCADA system works on a huge network like a smart grid, so it is difficult to make an attack tree or attack graph for the sophisticated vulnerability quantification because of numerous subdevices.

This survey shows the unsuitability of the existing schemes and the need for a common network model of the IEC 61850-based SAS. This gap provides an opportunity for the existing verified vulnerability quantification schemes to apply to IEC 61850-based SASs.

\section{IEC 61850-Based SAS Network Architecture}

Depending on the function of the elements, an IEC 61850based SAS can be classified into 3 levels [10], [11].

- Station Level: This level covers the overall management, observation, security, and control of substations. Well-known station-level devices include humanmachine interfaces (HMI), control system servers, and SNTP servers.

- Bay Level: This level receives data from process-level devices and takes charge of the inspection, security, control, measurement, and security breach prevention of bay-level devices. Well-known bay-level devices are protection IEDs, control IEDs, and monitors.

- Process Level: This level measures the physical information (electric current, voltage, temperature, etc.) or controls the movements of the machine. Devices that belong to this level include the current transformer, potential transformer, sensor, merging unit, and actuator.

The communication between each level occurs through a communication bus, and communication between the bay and station levels is designed based on an Ethernet with the IEC 61850 standard. Therefore, all IEDs are controlled and managed through HMI or by a substation controller through an existing substation protocol such as GOOSE, MMS, or SV.

Figure 2 shows the SAS network architecture in IEC 61850-7-1 and IEC 62351-10.

\section{Proposed Network Modeling and Evaluation Ap- proach for Security Vulnerability Quantification}

To quantify security vulnerabilities with IEC 61850-based SASs, it is necessary to simplify the network structure. That 

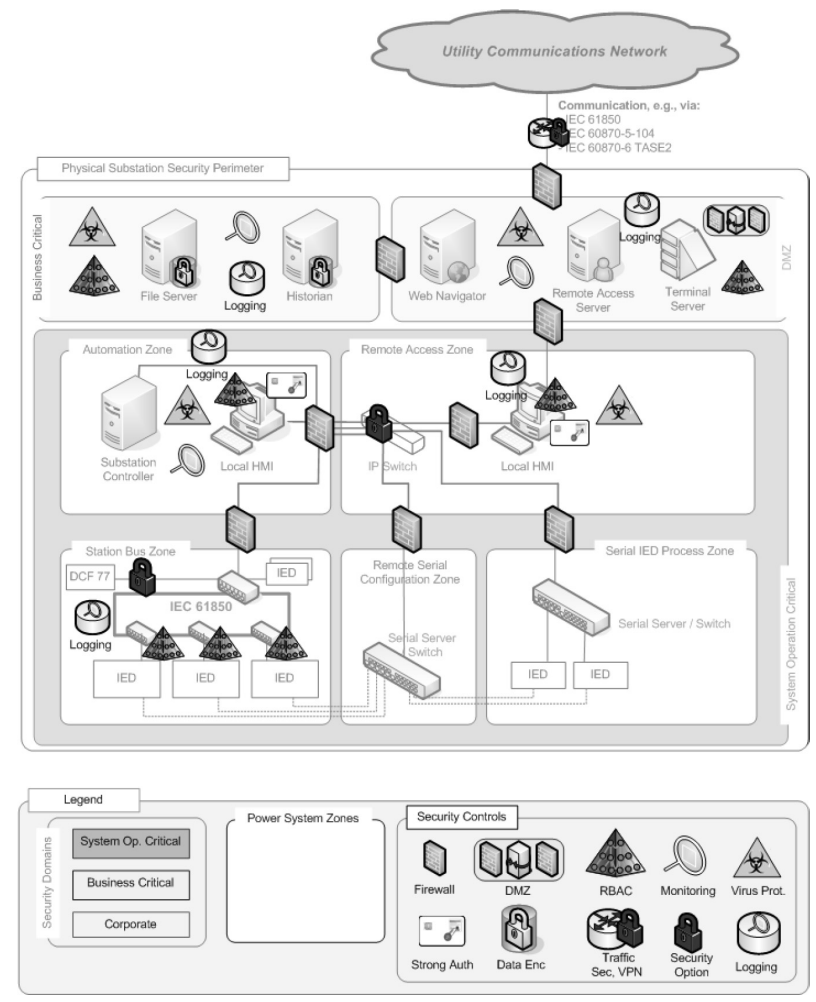

Fig. 2 SAS network architecture based on IEC 61850-7-1 and IEC 62351-10 [10], [11].

is, the network structure consists of many of the same devices that have the same functionalities, and from the security vulnerability quantification perspective, this does not influence the result. Hence, we propose a network modeling approach based on a simplified network structure, and we propose attack scenarios based on the multiple attack routes using zoning scheme to evaluate the proposed network model.

\subsection{IEC 61850-Based Network Modeling with Level and Device Group}

- Level-based Network Modeling

We simplified the level structure with the following device grouping according to the high accessibility by an attacker. The process-level devices have relatively lower accessibility and vulnerability influence than station- and baylevel devices. Therefore, our network modeling approach only covers the station and bay levels.

- Device Grouping

To audit an integrated environment of IEC 61850-based network devices such as IEDs, switches, and firewalls, the device grouping process is based on the characteristic of the devices in each level. The IED groups are categorized based on their tasks and recategorized based on their manufacturer characteristics. Then, other network devices (switches, firewalls, substation controllers, etc.) are categorized by their characteristics.

IED groups in the proposed model are grouped by their

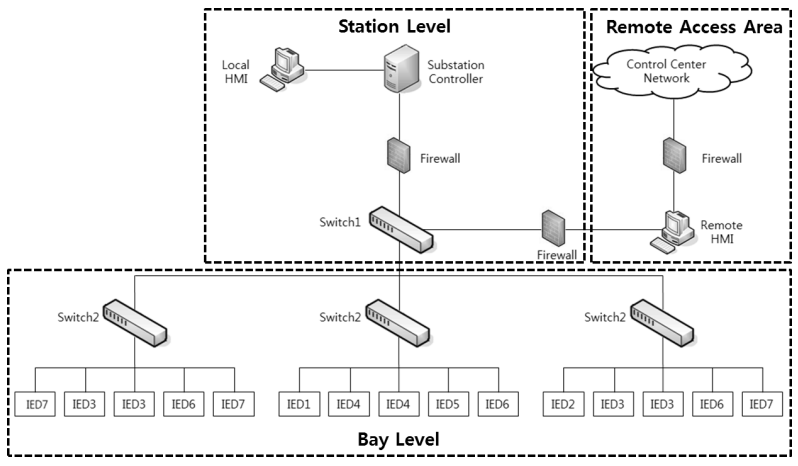

Fig. 3 Proposed IEC 61850-based SAS network model.

function/role in the substation. We assumed that Group 1 (IEDs 1, 2, 3, 4) contains the IEDs that serve as protection relay, Group 2 (IEDs 5, 6) contains the IEDs that serve as line protection and control, and Group 3 (IED 7) contains the IEDs that serve as a different protection relay than Group 1. Other firewalls, substation controllers, switches, and HMIs are assumed to follow the general SAS standard.

The number of the switches (Switch_1, Switch_2) is assigned depending on the role, not their unique number. The role of Switch 2 is to collect data from low-level IEDs, and Switch_1 collects data from multiple Switch_2s and sends them to the station level. The three IEDs from Switch 2 in Fig. 3 are numbered according to their unique characteristics and functions. IEDs may be numbered identically and perform the same task, but if they handle different data, they belong to different switches. Thus, an IED number is not a unique number like a serial device number.

- Proposed Network Modeling Approach

Figure 3 shows the proposed IEC 61850-based SAS network model for security vulnerability quantification. We classified the station and bay levels by applying the device groupings on IEDs. We also provide remote access areas considering access from an external connection.

\subsection{IEC 61850-Based Network Evaluation Using Attack Scenarios}

- Zoning

To create an attack scenario, some prior work is necessary to set the network zone according to the firewall locations. We used a firewall as the yardstick, because attacks that can penetrate security devices and those that cannot have different attack patterns. Hence, the exterior network of a substation is set as Zone 1, the remote HMI zone is set as Zone 2, and the zone with the IED devices is set as Zone 3.

By zoning, we can categorize the attack type on the proposed network model. If the attacks are restricted to a single zone, we call this "Penetrate." Such attacks might include obtaining the root privilege or infecting the system. Second, attacks permeating from one zone to other zones are called "Breach." These attacks use the devices in intermediate zones as intermediaries to the target zone. 


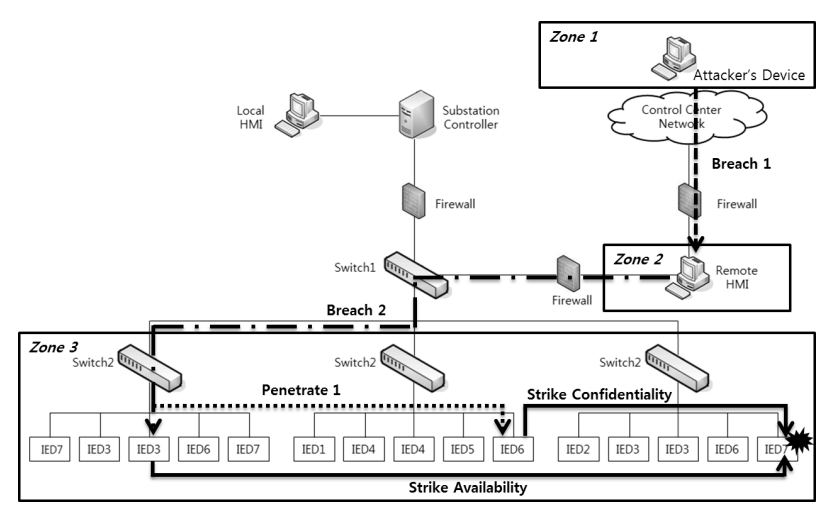

Fig. 4 Attack scenarios on proposed network model.

- Attack Scenarios

In Fig. 4, the attacker's computer is in Zone 1 and the ultimate target is the IED 7, which is located in the rightmost part of Zone 3. The attack proceeds in the unit of the zone and the attacker starts the attack from Zone 1 to IED 7 of Zone 3 by going through Zone 2. The attacker tries to breach Zone 2 using the vulnerability of the remote HMI, which is positioned in Zone 2. Further, using the vulnerability of IED 3 in Zone 3, the attacker can breach Zone 3 and obtain the ability to attack directly from IED 3 to IED 7. It is also possible to attack the confidentiality of IED 7 using the vulnerabilities of other IEDs (IED 6) that are in the same zone as IED 3 (Zone 3). This means that all devices in the same zone have the potential of being used in the attack scheme.

Attacks do not always have to start from Zone 1. Attacks may start from an infected computer in Zone 2, a malicious insider in Zone 3, or other zones that are not provided in this simplified attach scenario of the network model.

\section{Experimental Results and Analysis}

To verify a security vulnerability quantification suitability of the proposed network modeling with attack scenarios, we experimented with our network model by applying the actual security vulnerability quantification scheme.

We applied the MTTC [12] scheme for security vulnerability quantification on the proposed network model and attack scenarios. Unlike CVSS, the result of MTTC shows the attack success time according to multiple attack routes and attacker skill level, so it offers the security administrator more direct information, and it also relies less on CVE [2].

A number of existing vulnerabilities by the attack routes are important for calculating MTTC. Table 1 shows the number of vulnerabilities on proposed network model obtained by vulnerability scanning tools such as NMAP and NESSUS.

MTTC is the average value of multiple TTCs by attack routes. It is possible to obtain more accurate data from MTTC by applying the attacker's skill level. Figure 5 shows the result of MTTC calculation on the proposed network model with attack scenarios. Because it shows the mini-

\begin{tabular}{cc} 
Table 1 & Number of vulnerabilities. \\
\hline Target & Number of Vulnerabilities \\
\hline $\begin{array}{c}\text { Remote } \\
\text { HMI }\end{array}$ & 5 (Breach) \\
\hline $\begin{array}{c}\text { IED } \\
1-4\end{array}$ & 3 (Breach) \\
\hline IEC & 4 (Penetrate) \\
$5-6$ &
\end{tabular}

\begin{tabular}{|l|l|}
\hline Expert & 14.32 \\
\hline Intermediate & 13.2 18.8 \\
\hline Beginner & 16.0720 .82 \\
\hline
\end{tabular}

Fig. 5 MTTC intervals.

mum and maximum of the TTC for calculation of MTTC, it is called MTTC intervals.

Each bar shows the MTTC interval that represents the number of days spent on an attack. The attacker's expertise is calculated as the minimum TTC (13.2 days) and maximum TTC (18.8 days) according to two attack routes.

By adding every result obtained through applying probability of possible attack routes $(32 / 40,8 / 40)$ on each calculated TTC, we find that 14.32 days is the ultimate MTTC value. In the same way, the intermediate attacker's MTTC is calculated as 17.02 days and the beginner attacker's MTTC is calculated as 34.186 days.

With this evaluation, we can verify that it is possible to use the proposed network modeling and attack scenarios to quantify the actual security vulnerability.

\section{Conclusion}

IEC 61850-based SAS is a new research domain that includes complex network characteristics, so naturally its security standards, the IEC 62351 series, are still developing. Thus, it is not simple to create a security strategy for an SAS. In the Smart Grid security domain, security vulnerability quantification is a meaningful approach to increase the security of a target, but the characteristics of network diversity of IEC 61850-based SAS make its application difficult. In this paper, we proposed a novel network modeling approach for security vulnerability quantification for IEC 61850-based SASs. It includes network simplification using network-level and device groupings based on the functionality of IEDs. In addition, we presented attack scenarios that involved multiple attack routes with a zoning scheme. For the evaluation, we applied an MTTC scheme to the proposed approach. The evaluation results show the suitability of the proposed approach to security vulnerability quantification for IEC 61850-based SAS network. They also show that the proposed network modeling approach and attack scenarios might be applied to an actual SAS environment with a particular auditing scheme. In an actual environment, the secu- 
rity vulnerability quantification should be applied to every device and communication route on the network, not only to IED 7, to improve the quantification accuracy. Future work would consider the rest of the smart grid networks.

\section{Acknowledgments}

This work was supported by a Power Generation \& Electricity Delivery of the Korea Institute of Energy Technology Evaluation and Planning (KETEP) grant funded by the Korean Ministry of Knowledge Economy (No. 201210105000B).

\section{References}

[1] P. Mell, K. Scarfone, and S. Romanosky, "A Complete Guide to the Common Vulnerability Scoring System Version 2.0," Forum of Incident Response and Security Teams, 2007.

[2] P. Mell and T. Grance, Use of the Common Vulnerabilities and Exposures (CVE) Vulnerability Naming Scheme, NIST, 2002.

[3] NVD, "US National Vulnerability Database," http://nvd.nist.gov/, Feb. 2011. H. Holm, T.S

[4] IEC Std. 61850-1, "Introduction and overview," International Electro technical Commission, 2012.

[5] U. Premaratne, et al., "Security analysis and auditing of IEC61850- based automated substations," IEEE Trans. Power Deliv., vol.25, no.4, pp.2346-2355, 2010.

[6] M. Hentea, "Improving security for SCADA control systems," Interdisciplinary J. Information, Knowledge, and Management vol.3, no.1, pp.73-86, 2008.

[7] A. Hahn, "Smart grid architecture risk optimization through vulnerability scoring," 2010 IEEE Conference on Innovative Technologies for an Efficient and Reliable Electricity Supply (CITRES), 2010.

[8] J.L. Bayuk and A. Mostashari, "Measuring cyber security in intelligent urban infrastructure systems," 8th International Conference \& Expo on Emerging Technologies for a Smarter World (CEWIT), 2011.

[9] J. Yu, A. Mao, and Z. Guo, "Vulnerability assessment of cyber security in power industry," Power Systems Conference and Exposition, 2006. PSCE '06. 2006 IEEE PES. IEEE, 2006.

[10] IEC Std. 61850-7-1, "Communication networks and systems in substations Part 7-1: Basic communication structure for substation and feeder equipment. Principles and models," International Electro technical Commission, 2012.

[11] IEC Std. 62351-10, "Power systems management and associated information exchange - Data and communications security - Part 10: Security architecture guidelines," International Electro Technical Commission, Oct. 2012.

[12] D.J. Leversage and E. James, "Estimating a system's mean timeto-compromise," IEEE Security \& Privacy, vol.6, no.1, pp.52-60, Jan.-Feb. 2008. 